\title{
Molecular analysis of the carbapenem and metronidazole resistance mechanisms of Bacteroides strains reported in a Europe-wide antibiotic resistance survey
}

\author{
József Sóki*, Zsuzsa Eitel, Edit Urbán, Elisabeth Nagy, on behalf of the ESCMID Study Group on Anaerobic \\ Infections $^{1}$
}

Institute of Clinical Microbiology, Faculty of Medicine, University of Szeged, Semmelweis 6, H-6725 Szeged, Hungary

\section{A R T I C L E I N F O}

Article history:

Received 8 September 2012

Accepted 3 October 2012

\section{Keywords:}

Antibiotic resistance mechanisms

Bacteroides fragilis

cfiA

nim

Insertion sequence (IS) elements

\begin{abstract}
A B S T R A C T
Here we examine the carbapenem and metronidazole resistance mechanisms of 640 Bacteroides strains reported in the 2008-2009 European antibiotic susceptibility survey. Of the 22 strains with elevated imipenem minimum inhibitory concentrations $(\geq 4 \mu \mathrm{g} / \mathrm{mL}$ ), 10 were $c f i A$-positive and out of these 5 carried activating insertion sequence (IS) elements in the upstream regions of the cfiA genes. However, resistant strains with cfiA genes but with no activating IS elements were found $(n=2)$ as well as a resistant strain with no cfiA gene. In the former the resistance phenotypes by Etest were heterogeneous, whilst in the latter no carbapenemase production was seen; both mechanisms have been rarely observed, examined and characterised. Interestingly, few $(n=3)$ nim-positive strains were found, including one metronidazole-resistant strain harbouring nimE activated by ISBf6, and two susceptible strains harbouring chromosomally located nim genes.
\end{abstract}

(C) 2012 Elsevier B.V. and the International Society of Chemotherapy. All rights reserved.

\section{Introduction}

Bacteroides spp. represent one of the most significant groups of anaerobic bacteria. They are important constituents of the intestinal microbiota, from where they can cause severe anaerobic infections ranging from those of the soft tissue and upper respiratory tract to sepsis and various abscesses [1]. Bacteroides spp. can harbour the highest number of antibiotic resistance mechanisms and have the highest antibiotic resistance prevalences among all pathogenic anaerobes [2]. Because of their special and usually long culture requirements, temporary records of antibiotic resistance rates is considered a good and recommended practice worldwide. Such monitoring was performed mostly in the USA [3] and Europe [4], the latter under the organisation of the European Society of Clinical Microbiology and Infectious Diseases (ESCMID) Study Group for Antimicrobial Resistance in Anaerobic Bacteria (ESGARAB), whose name was changed to the ESCMID Study Group on Anaerobic Infections to cover a broader interest. The general

\footnotetext{
* Corresponding author. Tel.: +36 62545 712; fax: +36 62545712.

E-mail address: soki.jozsef@med.u-szeged.hu (J. Sóki).

1 ESCMID Study Group on Anaerobic Infections: Denis Piérard, Youri Glupczynski, Claire Nonhoff, Margareta Ieven (Belgium); Smilja Kalenič (Croatia); Eva Chmelařova (Czech Republic); Eija Können (Finland); Laurent Calvet, Luc Dubreuil (France); Erika Dósa (Germany); Joseph Papaparaskevas (Greece); Lenke Szikra, Cecilia Miszti, Gabriella Terhes (Hungary); Annarita Mazzariol (Italy); Jordi Vila (Spain); Maria Hedberg (Sweden); John Degener, Linda Wildeboer-Veloo (The Netherlands); and Nezhat Güler, Sabiha Güner, Nurver Ulger (Turkey).
}

trend is almost $100 \%$ resistance to penicillins, cephalosporins and tetracycline, a rising moderate resistance prevalence to cefoxitin, clindamycin and moxifloxacin, and very low prevalences for carbapenems, $\beta$-lactam/ $\beta$-lactamase combinations, metronidazole and tigecycline $[3,4]$. Following antibiotic resistance monitoring for Bacteroides in 2000, molecular analyses were carried out to determine the metronidazole and carbapenem resistance mechanisms [5,6]. These investigations demonstrated the roles of the nim and cfiA genes and their activating insertion sequence (IS) elements in metronidazole and carbapenem resistance mechanisms, respectively.

Carbapenem-resistant Bacteroides isolates usually belong to the Bacteroides fragilis group, with the cfiA resistance gene being chromosomal and the majority of $c f i A$-positive strains being susceptible phenotypically because of the lack of upregulating IS elements [1]. The best-characterised metronidazole resistance mechanism among Bacteroides strains is due to the $\operatorname{nim}$ genes $(\operatorname{nim} A-F)$ that may occur in all Bacteroides species, and they are either located on well-characterised plasmids or on the chromosome. The majority of nim-positive Bacteroides isolates studied harbour a nim gene and a corresponding IS element pair [6]. It is of interest that the cfiA-positive $B$. fragilis isolates form a subgroup within this species. The $c f i A$-negative and $c f i A$-positive strains are therefore often classified as Division I and II, respectively, and can be distinguished by differences in DNA-DNA homology rates and by molecular typing methods such as randomly amplified polymorphic DNA polymerase chain reaction (RAPD-PCR), ribotyping, multilocus enzyme electrophoresis, sequence typing and

0924-8579/\$ - see front matter (C) 2012 Elsevier B.V. and the International Society of Chemotherapy. All rights reserved. http://dx.doi.org/10.1016/j.ijantimicag.2012.10.001 
Table 1

Primers and PCR conditions originally designed and applied in this study.

\begin{tabular}{|c|c|c|c|}
\hline PCR & Primers & Sequence $5^{\prime} \rightarrow 3^{\prime}$ & PCR conditions \\
\hline \multirow[t]{2}{*}{ cfiA } & cfiA-RT1 & AATCGAAGGATGGGGTATGG & \multirow[t]{2}{*}{$95^{\circ} \mathrm{C}$ for $5 \mathrm{~min}$; 35 cycles of $95^{\circ} \mathrm{C}$ for $15 \mathrm{~s}, 59^{\circ} \mathrm{C}$ for $1 \mathrm{~min}, 72^{\circ} \mathrm{C}$ for $30 \mathrm{~s}$; melting $72-95^{\circ} \mathrm{C}$} \\
\hline & cfiA-RT2 & CGGTCGGTGAATCGGTGAAT & \\
\hline \multirow[t]{2}{*}{$\operatorname{nim}^{\mathrm{a}}$} & $\operatorname{nim} 3$ & ATGTTCAGAGAAATGCGGCGTAAGCG & \multirow[t]{2}{*}{$95^{\circ} \mathrm{C}$ for $10 \mathrm{~min}$; 35 cycles of $95^{\circ} \mathrm{C}$ for $30 \mathrm{~s}, 62^{\circ} \mathrm{C}$ for $1 \mathrm{~min}, 72^{\circ} \mathrm{C}$ for $1 \mathrm{~min}$; melting $72-95^{\circ} \mathrm{C}$} \\
\hline & $\operatorname{nim} 5$ & GCTTCCTTGCCTGTCATGTGCTC & \\
\hline
\end{tabular}

a The method of Trinh and Reysset was adapted to real-time PCR [13].

matrix-assisted laser-desorption/ionisation time-of-flight (MALDITOF) mass spectrometry [7-12]. The Ambler class A cephalosporinase gene, серA, and the enterotoxin bft genes were reported to occur exclusively in Division I strains [10].

This study investigated the prevalences of the cfiA and nim genes, the imipenem and metronidazole resistance mechanisms in the majority of Bacteroides strains reported in the 2008 European Bacteroides antibiotic resistance survey.

\section{Materials and methods}

\subsection{Bacterial strains and cultivation}

A total of 640 isolates belonging to the Bacteroides and Parabacteroides genera (486 B. fragilis, 54 Bacteroides thetaiotaomicron, 36 Bacteroides ovatus, 33 Bacteroides vulgatus, 8 Bacteroides uniformis, 7 Parabacteroides distasonis, 4 Parabacteroides merdae, 3 Bacteroides eggerthii, 3 Bacteroides massiliensis, 3 Bacteroides nordii, 2 Bacteroides caccae and 1 Bacteroides stercoris) were analysed from the collection sent to the central laboratory (Institute of Clinical Microbiology, University of Szeged, Szeged, Hungary) for the 2008-2009 European Bacteroides antibiotic susceptibility survey (participating countries: Belgium, Croatia, Czech Republic, Finland, France, Germany, Greece, Hungary, Italy, Spain, Sweden, The Netherlands and Turkey). Isolate identification was carried out by routine clinical methods. Strains were stored at $-70^{\circ} \mathrm{C}$ in CryoBank vials (Mast Diagnostica, Rheinfeld, Germany) and were cultivated at $37{ }^{\circ} \mathrm{C}$ anaerobically on Columbia agar supplemented with $5 \%(\mathrm{v} / \mathrm{v})$ sheep blood, $5 \mathrm{~g} / \mathrm{L}$ haemin and $1 \mathrm{~g} / \mathrm{L}$ vitamin $\mathrm{K}_{1}$, or in BHIS broth [brain-heart infusion broth supplemented with $0.5 \%(\mathrm{w} / \mathrm{v})$ yeast extract, $5 \mathrm{~g} / \mathrm{L}$ haemin and $1 \mathrm{~g} / \mathrm{L}$ vitamin $\mathrm{K}_{1}$ ] in an anaerobic cabinet (Concept 400; Ruskinn Technology Ltd., Bridgend, UK) under a gas composition of $85 \% \mathrm{~N}_{2}, 10 \% \mathrm{H}_{2}$ and $5 \% \mathrm{CO}_{2}$ for $48 \mathrm{~h}$. Antibiotic resistance results were obtained from the susceptibility measurements done previously by the agar dilution method [4] or by Etest (bioMérieux, Marcy-l'Étoile, France) as recommended by the supplier. The following control strains were used: B. fragilis TAL3636 (cfiA); B. fragilis 638R (pIP417) (nimA); B. fragilis BF-8 (nimB); B. fragilis 638R (pIP419) (nimC); B. fragilis 638R (pIP421) (nimD); and $B$. fragilis 388 (nimE).

\subsection{Real-time PCR detection of the cfiA and nim genes}

Bacterial template DNA samples for the real-time PCR analysis were prepared by incubating $100 \mu \mathrm{L}$ of $0.5 \mathrm{McF}$ arland turbidity suspensions at $100^{\circ} \mathrm{C}$ for $10 \mathrm{~min}$, which were stored at $-30^{\circ} \mathrm{C}$ until use. Real-time PCR experiments were carried out in an MXPro3000 instrument (Stratagene, Santa Clara, CA) with the following reaction setup: $1 \times$ MasterMix [ $\mathrm{iQ}^{\mathrm{TM}}$ (Bio-Rad Hungary, Budapest, Hungary) with $1 \times$ EvaGreen $^{\circledR}$ (Biotium Inc., Hayward, CA) for nim; or Brilliant III (Stratagene/Agilent, Santa Clara, CA) for cfiA and $b f t$ ], $0.7 \mu \mathrm{M}$ of each primer and $2 \mu \mathrm{L}$ of template DNA preparation in $10 \mu \mathrm{L}$ final volumes in 96-well PCR reaction plates. The nucleotide sequences of the newly used primers and the cycling conditions chosen during this study are shown in Table 1 . Positive reactions were identified by the starting amplification cycle, melting curves showing the correct melting temperatures, and in rare cases where it was required to compare the size of the products with those of the positive controls in $1.2 \%$ agarose gel electrophoresis.

Table 2

Analysis of the imipenem resistance mechanism of strains with elevated imipenem minimum inhibitory concentrations (MICs) ( $\geq 4 \mu \mathrm{g} / \mathrm{mL}$ ).

\begin{tabular}{|c|c|c|c|c|}
\hline Strain & Imipenem MIC $(\mu \mathrm{g} / \mathrm{mL})$ & cfiA & Upstream region & Mechanism \\
\hline Bacteroides fragilis SW42 & 4 & - & - & Other ${ }^{\mathrm{a}}$ \\
\hline B. fragilis SW46 & 4 & - & - & Other \\
\hline B. fragilis SW83 & 4 & - & - & Other \\
\hline B. fragilis TR38 & 4 & - & - & Other \\
\hline B. fragilis HU25 & 4 & - & - & Other \\
\hline B. fragilis FI63 & 4 & - & - & Other \\
\hline Bacteroides eggerthii GR67 & 4 & - & - & Other \\
\hline Bacteroides thetaiotaomicron BEM28 & 4 & - & - & Other \\
\hline Parabacteroides merdae GR70 & 4 & - & - & Other \\
\hline B. fragilis DE14 & 4 & + & $280 \mathrm{bp}^{\mathrm{b}}$ & Silent with increased MIC \\
\hline B. fragilis HU51 & 4 & + & $280 \mathrm{bp}^{\mathrm{b}}$ & Silent with increased MIC \\
\hline B. fragilis IT15 & 4 & + & IS4351 & IS-activated \\
\hline Bacteroides stercoris HU59 & 8 & - & - & Other \\
\hline B. thetaiotaomicron BEA22 & 8 & - & - & Other \\
\hline B. fragilis HU92 & 8 & + & $280 \mathrm{bp}^{\mathrm{b}}$ & Silent with increased MIC \\
\hline B. fragilis TR27 & 16 & + & IS1187 & IS-activated \\
\hline B. fragilis TR31 & 16 & + & IS1187 & IS-activated \\
\hline B. fragilis HU61 & 32 & + & $280 \mathrm{bp}^{\mathrm{b}}$ & Heteroresistant \\
\hline B. fragilis NLH3 & $>32$ & + & ISBf11 & IS-activated \\
\hline B. fragilis FR41 & $>32$ & + & $280 \mathrm{bp}^{\mathrm{b}}$ & Heteroresistant \\
\hline B. fragilis FI87 & $>32$ & + & IS614B & IS-activated \\
\hline B. fragilis FI37 & $>32$ & - & - & Other \\
\hline
\end{tabular}

a The effects are not caused by cfiA.

b The 280-bp PCR fragment displays no insertion upstream of cfiA. 


\subsection{Analysis of the carbapenem and metronidazole resistance mechanisms by molecular methods}

An analysis of the carbapenem and metronidazole resistance mechanisms was carried out as previously described $[14,15]$. Imipenemase activities were recorded in a $50 \mathrm{mM} \mathrm{NaPO}_{4}(\mathrm{pH}$ 7.0) buffer using sonicated cell extracts and $0.1 \mathrm{mM}$ imipenem by following absorbance changes at $299 \mathrm{~nm}$. Protein concentrations were measured with a Quant-iT ${ }^{\mathrm{TM}}$ Protein Assay Kit using a Qubit ${ }^{\circledR}$ Mini Fluorometer (Life Technologies Hungary Ltd., Budapest, Hungary). Tazobactam $(10 \mu \mathrm{g} / \mathrm{mL})$ or $10 \mathrm{mM}$ ethylene diamine tetra-acetic acid (EDTA) were used to inhibit the enzymes, and imipenemase activity was expressed as $1 \mathrm{nmol}$ hydrolysed imipenem/min ( $1 \mathrm{U}$ ) standardised by the protein concentration of the sonicates. Nucleotide sequencing was performed using an automated sequencer as described previously [15]. The novel nucleotide sequence of ISBf 11 was deposited in the GenBank database under accession no. GQ449386.

\section{Results and discussion}

\subsection{Resistance mechanisms of Bacteroides strains with elevated imipenem minimum inhibitory concentrations (MICS)}

Of the 640 Bacteroides strains included in this study, 22 had imipenem MICs $\geq 4 \mu \mathrm{g} / \mathrm{mL}$. Of the 486 B. fragilis strains examined, 43 were cfiA-positive, and from the 640 Bacteroides isolates examined 22 and 7 had imipenem MICs $\geq 4 \mu \mathrm{g} / \mathrm{mL}$ and $\geq 16 \mu \mathrm{g} / \mathrm{mL}$, respectively. No non-fragilis Bacteroides strains were resistant to imipenem and only one cfiA-negative $B$. fragilis isolate was resistant. The results are summarised in Table 2 . Of the $10 \mathrm{~B}$. fragilis strains with elevated imipenem MICs $(4-8 \mu \mathrm{g} / \mathrm{mL}), 4$ (40.0\%) were $c f i A$-positive, whilst $6(85.7 \%)$ of the 7 imipenem-resistant (MIC $\geq 16 \mu \mathrm{g} / \mathrm{mL}$ ) B. fragilis isolates were cfiA-positive. Among the strains with elevated MICs and with cfiA genes, one harboured an IS element upstream of $c$ fiA (B. fragilis IT15), and among the cfiA-positive and imipenem-resistant strains four harboured IS elements upstream of the resistance gene (Table 2). The remaining two cfiA-positive isolates that were imipenem-resistant but without activating IS elements upstream of cfiA displayed a heterogeneous resistance phenotype using the imipenem Etest (see the example in Fig. 1).

This study yielded similar prevalence values for the molecular mechanisms of imipenem resistance of $B$. fragilis strains as those in previous studies. Among the highly imipenem-resistant strains $(\mathrm{MIC} \geq 16 \mu \mathrm{g} / \mathrm{mL}$ ), the cfiA genes are activated by IS elements ( 4 of 6 cfiA-positive), and among strains with elevated imipenem MICs $(\geq 4 \mu \mathrm{g} / \mathrm{mL})$ the $c f i A$ genes were enriched $(26.7 \%$ compared with the commonly found $2-8 \%$ ). The types of $c f i A$-activating IS elements were IS1187 $(n=2)$, IS614B $(n=1)$, and a novel IS element ( $n=\operatorname{ISBf11}$; GenBank accession no. GQ449386) for B. fragilis H3 that had 77\% homology compared with IS614B (Table 2). Bacteroides fragilis IT15 harboured IS4351 upstream of the cfiA gene, but its imipenem MIC was low $(4 \mu \mathrm{g} / \mathrm{mL})$. This latter finding is in accordance with that of Podglajen et al. [8] who found that $B$. fragilis strains carrying IS4351 upstream of the cfiA genes also tended to have low imipenem MICs $(16 \mu \mathrm{g} / \mathrm{mL})$ compared with other IS elements (IS942 and IS1186; MICs $\geq 64 \mu \mathrm{g} / \mathrm{mL}$ ). Previously we detected a probably low-activity Bacteroides promoter-like sequence in the upstream regions of the cfiA genes. According to this, our study and other studies detected elevated imipenemase activities in 'silent' cfiA-positive strains that could account for the imipenem MICs in such strains [14,16]. However, some highly imipenem-resistant strains $(n=2)$ were also genetically silent, their cfiA genes not being activated by IS elements (Table 2). In these cases Etest susceptibility tests detected

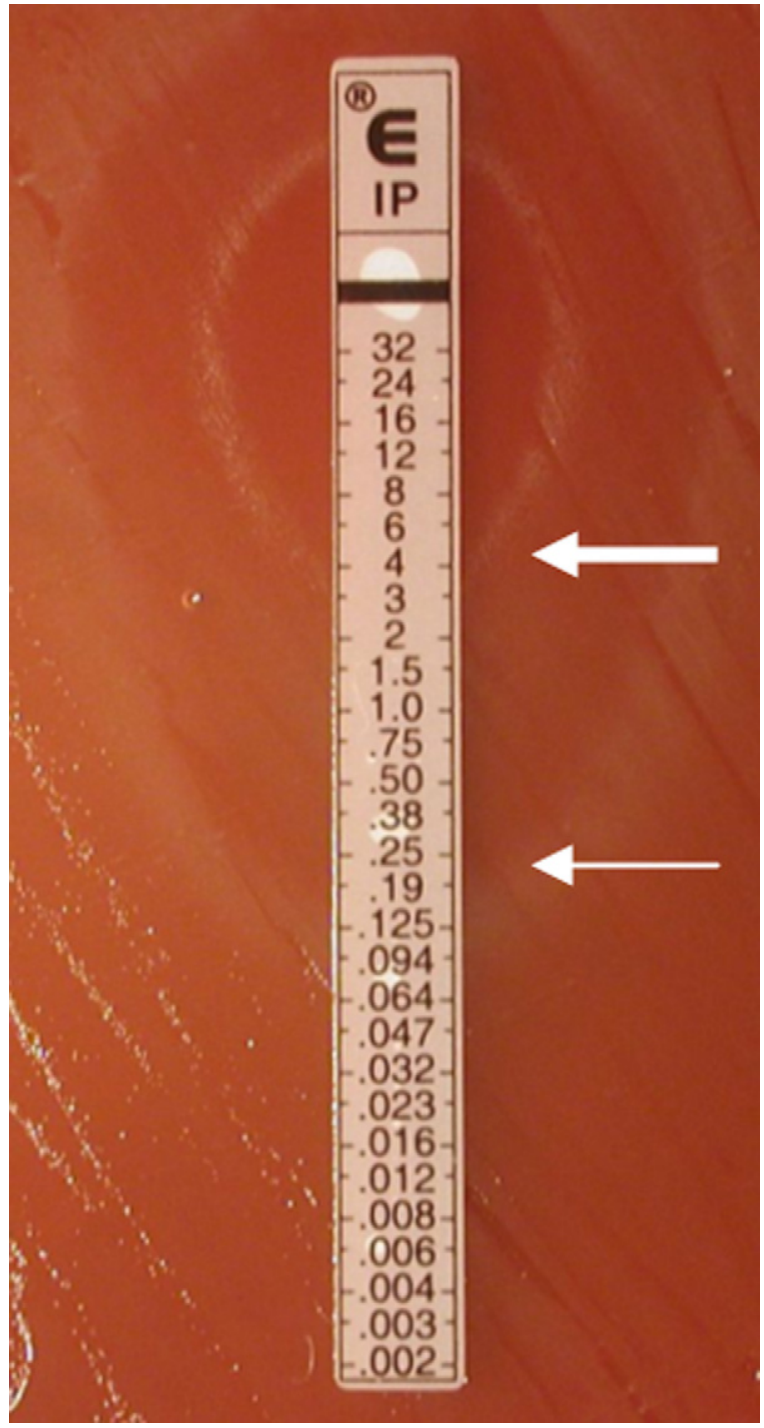

Fig. 1. Heterogeneous imipenem-resistant phenotype of Bacteroides fragilis FR41 detected by Etest. The first $(0.25 \mu \mathrm{g} / \mathrm{mL})$ and second $(4 \mu \mathrm{g} / \mathrm{mL})$ inhibition zones are marked by thin and thicker arrows, respectively.

heterogeneous resistance phenotypes (Fig. 1) where, inside of confluent inhibition zones, resistant colonies or growth appeared. This phenomenon can be explained by activation of the cfiA genes by an as yet unidentified mechanism that boosts the carbapenemase activity of the strains. We previously described such heteroresistant strains from human faeces whose imipenem MICs and imipenemase activities displayed a relation. In contrast, the $c f i A$ genes were not activated by IS elements [16]. For Bacteroides, we detected heterogeneously cefoxitin-resistant strains and hypothesised that the copy number of the corresponding $c f x A$ resistance gene might be important [17].

A cfiA-negative but imipenem-resistant $B$. fragilis isolate was identified in this study (B. fragilis FI37; Table 2). Such strains were also found previously, but the exact carbapenem resistance mechanism for these strains has not yet been clarified $[18,19]$. An imipenemase assay of this strain did not reveal any activity, whilst the control B. fragilis TAL3636 strain produced $41.0 \mathrm{U} / \mathrm{mg}$ imipenemase activity that was inhibited by EDTA. The probable resistance mechanisms are penicillin-binding protein (PBP) affinity or permeability changes.

A strain-dependent role for PBPs in the case of eight $B$. fragilis strains with various imipenem MICs $(0.12-16 \mu \mathrm{g} / \mathrm{mL})$ was reported 
previously [19], and mutations of an endogenous efflux system (bmeABC) also affected the carbapenem susceptibilities of the carrying strains [20].

\subsection{Detection of nim genes and their relation to metronidazole resistance}

Of the 640 Bacteroides strains, 21 had reduced susceptibility to metronidazole (MIC $\geq 4 \mu \mathrm{g} / \mathrm{mL}$ ) and only 3 (B. fragilis IT724 and IT797 and B. thetaiotaomicron HU66) harboured nim genes, with the following metronidazole MICs: $0.125 \mu \mathrm{g} / \mathrm{mL}$ (B. fragilis IT797), $1 \mu \mathrm{g} / \mathrm{mL}$ (B. fragilis IT724) and $256 \mu \mathrm{g} / \mathrm{mL}$ (B. thetaiotaomicron HU66). An examination of the nim-mediated resistance mechanisms revealed that $B$. fragilis IT797 and IT724 harboured chromosomal nimA and nim $C$ genes, respectively. By contrast, the nimE gene of $B$. thetaiotaomicron HU66 was located on an $8.3 \mathrm{~kb}$ (pBF388c-like) [15] plasmid and was activated by ISBf6 (data not shown). No nim-specific plasmids were detected in the two other strains (a $5.6 \mathrm{~kb}$ class III plasmid and no plasmid content were characteristic for B. fragilis IT797 and IT724, respectively). Furthermore, B. fragilis IT797 harboured IS1168 and IS1170, but these elements could not be mapped to the nimA gene by PCR mapping. From these results, it appears that the situation with nim-mediated metronidazole-resistant Bacteroides strains has changed in Europe compared with the previous study where 43 Bacteroides strains with reduced metronidazole susceptibility (MICs $\geq 4 \mu \mathrm{g} / \mathrm{mL} ; 3.3 \%$ ) and $30(2.0 \%)$ nim-positive strains were found [6]. The current situation in Europe is reminiscent of that in the USA where nim genes and metronidazole resistances were scarce for a long time [21]. The nim-negative but metronidazole-resistant Bacteroides strains found in the current study may have other resistance mechanisms (reduced uptake, nitroreductase and pyruvate-ferredoxin oxidoreductase activities, increased lactate dehydrogenase activity, or mutations that alter the carbohydrate utilisation affecting the redox state) which shortcut the detrimental cellular effects of this drug [2,22-24].

In conclusion, these results confirmed the present view of carbapenem and metronidazole resistance mechanisms of Bacteroides spp. but also provide new information regarding their current state and epidemiology in Europe in addition to newly described mechanisms such as non-carbapenemase-mediated imipenem resistance and chromosomal nim genes.

Funding: This study was supported by the Excellence Centre of the University of Szeged (Szeged, Hungary) (TÁMOP 421B), the Hungarian National Research Fund (OTKA 69044) and the ESCMID Study Group of Anaerobic Infections.

Competing interests: None declared.

Ethical approval: Not required.

\section{References}

[1] Wexler HM. Bacteroides: the good, the bad and the nitty-gritty. Clin Microbiol Rev 2007;20:593-621.

[2] Rasmussen BA, Bush K, Tally FP. Antimicrobial resistance in Bacteroides. Clin Infect Dis 1993;16(Suppl. 4):S390-400.
[3] Snydman DR, Jacobus NV, McDermott LA, Golan Y, Hecht DW, Goldstein EJC, et al. Lessons learned from the anaerobe survey: historical perspective and review of most recent data (2005-2007). Clin Infect Dis 2010;50(Suppl. 1):S26-33.

[4] Nagy E, Urbán E, Nord CE; ESCMID Study Group on Antimicrobial Resistance in Anaerobic Bacteria. Antimicrobial susceptibility of Bacteroides fragilis group isolates in Europe; 20 years of experience. Clin Microbiol Infect 2011;17:371-9.

[5] Sóki J, Edwards R, Hedberg M, Fang H, Nagy E, Nord CE. Examination of cfiAmediated carbapenem resistance in Bacteroides fragilis strains from a European antibiotic susceptibility survey. Int J Antimicrob Agents 2006;28:497-502.

[6] Löfmark S, Fang H, Hedberg M, Edlund C. Inducible metronidazole resistance and nim genes in clinical Bacteroides fragilis group isolates. Antimicrob Agents Chemother 2005;49:1253-6.

[7] Johnson JL. Taxonomy of the Bacteroides. I. Deoxyribonucleic acid homologies among Bacteroides fragilis and other saccharolytic Bacteroides species. Int J Syst Bacteriol 1978;28:245-56.

[8] Podglajen I, Breuil J, Casin I, Collatz E. Genotypic identification of two groups within the species Bacteroides fragilis by ribotyping and by analysis of PCRgenerated fragment patterns and insertion sequence content. J Bacteriol 1995; 177:5270-5.

[9] Ruimy R, Podglajen I, Breuil J, Christen R, Collatz E. A recent fixation of cfiA genes in a monophyletic cluster of Bacteroides fragilis is correlated with the presence of multiple insertion elements. J Bacteriol 1996;178:1914-8.

[10] Gutacker M, Valsangiacomo C, Piffaretti JC. Identification of two genetic groups in Bacteroides fragilis by multilocus enzyme electrophoresis: distribution of antibiotic resistance (cfiA, cepA) and enterotoxin ( $b f t$ ) encoding genes. Microbiology 2000;146:1241-54.

[11] Wybo I, De Bel A, Soetens O, Echahidi F, Vandoorslaer K, van Cauwenbergh $\mathrm{M}$, et al. Differentiation of cfiA-negative and cfiA-positive Bacteroides fragilis isolates by matrix-assisted laser desorption ionization-time of flight mass spectrometry. J Clin Microbiol 2011;49:1961-4.

[12] Nagy E, Becker S, Sóki J, Urbán E, Kostrzewa M. Differentiation of division I ( cfiAnegative) and division II (cfiA-positive) Bacteroides fragilis strains by matrixassisted laser desorption/ionization time of-flight mass spectrometry. J Med Microbiol 2011;60:1584-90.

[13] Trinh S, Reysset G. Detection by PCR of the nim genes encoding 5-nitroimidazole resistance in Bacteroides spp. J Clin Microbiol 1996;34:2078-84.

[14] Soki J, Fodor E, Hecht DW, Edwards R, Rotimi VO, Kerekes I, et al. Molecular characterization of imipenem-resistant cfiA-positive Bacteroides fragilis isolates from the USA, Hungary and Kuwait. J Med Microbiol 2004;53:413-9.

[15] Sóki J, Gal M, Brazier JS, Rotimi VO, Urbán E, Nagy E, et al. Molecular investigation of genetic elements contributing to metronidazole resistance in Bacteroides strains. J Antimicrob Chemother 2006;57:212-20.

[16] Sóki J, Edwards R, Urbán E, Fodor E, Beer Z, Nagy E. Screening of isolates from faeces for carbapenem-resistant Bacteroides strains; existence of strains with novel types of resistance mechanisms. Int J Antimicrob Agents 2004:24:450-4.

[17] Sóki J, Gonzalez SM, Urbán E, Nagy E, Ayala JA. Molecular analysis of the effector mechanisms of cefoxitin resistance among Bacteroides strains. J Antimicrob Chemother 2011;66:2492-500.

[18] Yamazoe K, Kato N, Kato H, Tanaka K, Katagiri Y, Watanabe K. Distribution of the cfiA gene among Bacteroides fragilis strains in Japan and relatedness of $c f i A$ to imipenem resistance. Antimicrob Agents Chemother 1999;43:2808-10.

[19] Ayala J, Quesada A, Vadillo S, Criado J, Píriz S. Penicillin-binding proteins of Bacteroides fragilis and their role in resistance to imipenem of clinical isolates. J Med Microbiol 2005;54:1055-64.

[20] Pumbwe L, Ueda O, Yoshimura F, Chang A, Smith RL, Wexler HM. Bacteroides fragilis $\mathrm{Bme} \mathrm{ABC}$ efflux systems additively confer intrinsic antimicrobial resistance. J Antimicrob Chemother 2006;58:37-46.

[21] Schapiro JM, Gupta R, Stefansson E, Fang FC, Limaye AP. Isolation of metronidazole-resistant Bacteroides fragilis carrying the nimA nitroreductase gene from a patient in Washington State. J Clin Microbiol 2004;42:4127-9.

[22] Narikawa S, Suzuki T, Yamamoto M, Nakamura M. Lactate dehydrogenase activity as a cause of metronidazole resistance in Bacteroides fragilis NCTC 11295. J Antimicrob Chemother 1991;28:47-53.

[23] Diniz CG, Farias LM, Carvalho MAR, Rocha ER, Smith CJ. Differential gene expression in a Bacteroides fragilis metronidazole-resistant mutant. J Antimicrob Chemother 2004;54:100-8.

[24] Patel EH, Paul LV, Casanueva AI, Patrick S, Abratt VR. Overexpression of rhamnose catabolism regulatory protein. RhaR: a novel mechanism for metronidazole resistance in Bacteroides thetaiotaomicron. J Antimicrob Chemother 2009;69:267-73. 